VOL. $3(1970), 363-368$.

\title{
Two properties of Bochner integrals
}

\author{
B. D. Craven
}

\begin{abstract}
Two theorems for Lebesgue integrals, namely the Gauss-Green Theorem relating surface and volume integrals, and the integration-by-parts formula, are shown to possess generalizations where the integrands take values in a Banach space, the integrals are Bochner integrals, and derivatives are Fréchet derivatives. For integration-by-parts, the integrand consists of a continuous linear map applied to a vector-valued function. These results were required for a generalization of the calculus of variations, given in another paper.
\end{abstract}

This paper assumes the definition, and standard properties, of Bochner integrals, as given in Hille and Phillips [2] and in Yosida [3]. Neither of these books gives the theorems proved in this note. Let $V$ denote a Banach space, over the real field, and let [V] denote the Banach space of all bounded linear maps from $V$ into $V$, with the usual norm. Let $I=[a, b]$ denote a compact real interval; let $X_{E}($. denote the characteristic function of $E$, where $E$ is a measurable subset of $I$.

Let $G$ be a bounded open subset of Euclidean $p$-space $R^{p}$, with boundary $\partial G$; let $\mu_{p}(x)$ denote $p$-dimensional Lebesgue measure, where $x=\left(x_{1}, \ldots, x_{p}\right) \in \mathrm{R}^{p}$; for $x \in \partial G$, let $v(x)$ and $\Phi(x)$ denote suitable defined unit exterior normal and surface area on the "surface" $\partial G$, as defined in Craven [1].

If $g(x)=\left(g_{1}(x), \ldots, g_{p}(x)\right)$ is a $p$-vector valued function of

Received 10 August 1970. 
$x$,

$$
\operatorname{div} g(x)=\sum_{i=1}^{p} \frac{\partial g_{i}(x)}{\partial x_{i}} \text { and } g(x) \cdot v(x)=\sum_{i=1}^{p} g_{i}(x) v_{i}(x)
$$

then the Gauss-Green Theorem states that, if $G$ and $g$ satisfy suitable conditions, then

$$
\int_{G} \operatorname{divg}(x) d \mu_{p}(x)=\int_{\partial G} g(x) \cdot \nu(x) d \Phi(x) .
$$

If $g$ maps the closure $\bar{G}$ of $G$ into $V^{p}$, where $V$ is a Banach space, instead of into $R^{p}$, and, for $i=1,2, \ldots, p, g_{i}$ is Fréchet-differentiable with respect to $x_{i}$, for fixed $x_{j}(j \neq i)$, then (1) and (2) remain meaningful in terms of Fréchet derivative and Bochner integrals; both $\operatorname{divg(.)}$ and $g(.) . v($.$) are maps of G$ into $V$. The Gauss-Green Theorem then holds in the following form:

THEOREM A. Let $G$ be a bounded open subset of $R^{p}$, such that $\partial G$ is a countable union of disjoint continuous images of the unit sphere in $\mathrm{R}^{p}$, and $\Phi(\partial G)<\infty$; let $E \subset G$ satisfy the some conditions as $\partial G$. Let $g: \bar{G} \rightarrow V^{p}$ be a continuous map, such that $\operatorname{divg}(x)$ exists at each point of $G-E$, and $\|$ aivg $(x) \|$ is Lebesgue integrable on $G$. Then (2) holds for $G, \partial G$, and $g$.

Proof. Since the proof differs only in a few key details from the proof for $V=R$ (Theorems $1, .2$ and 3 of [1]), only the changes need be stated. By Bochner's Theorem ([3], p. 133), integrability of \|divg $(x) \|$ implies that the left side of (2) exists as a Bochner integral. The proof of Theorem 1 of [1] remains applicable, with the norm $\|\cdot\|$ of $V$ replacing absolute value $|$.$| where appropriate. In the proof of Theorem$ 2 , equation (1I) applies with $\|$.$\| replacing |$.$| . The definition of$ the function $\psi$ requires modification. Let 


$$
\begin{aligned}
z=g_{i}\left(x_{1}, \ldots, x_{i-1}, b_{i},\right. & \left.x_{i+1}, \ldots, x_{p}\right) \\
& -g_{i}\left(x_{1}, \ldots, x_{i-1}, a_{i}, x_{i+1}, \ldots, x_{p}\right) \\
& -\int_{T_{i}} \frac{\partial g_{i}}{\partial x_{i}}\left(x_{1}, \ldots, x_{i-1}, x_{i}, x_{i+1}, \ldots, x_{p}\right) d x_{i} ;
\end{aligned}
$$

thus $z \in V$. Denote by $z^{\prime \prime}$ the canonical image of $z$ in the second dual space $V^{\prime \prime}$. Then Lemma 5 of [1] applies to

$$
w(x)=f\left(g_{i}\left(x_{1}, \ldots, x_{i}, x, x_{i+1}, \ldots, x_{p}\right)\right)
$$

for each $f$ in the dual space $V^{\prime}$ such that $\|f\|=1$. Then

$$
|f(z)| \leq K=N\left(b_{i}-a_{i}-\mu_{1}\left(T_{i}\right)\right) \text {, }
$$

so that

$$
\|z\|=\|z "\|=\sup \{|f(z)|:\|f\|=1\} \leq K .
$$

From this, equations (13) and (16) of the proof of Theorem 2 follow, with $\|\cdot\|$ replacing $|$.$| . Then Theorem 3$, with $g: \bar{G} \rightarrow V^{p}$ replacing $g: \bar{G}+R^{p}$, is an immediate consequence of the modified Theorems 1 and 2 . Integration by parts for the Bochner integral depends on the following lemma (for integration with respect to Lebesgue measure).

LEMMA 1. Let $f: I \rightarrow V$ be Bochner-integrable on $I$; let $T_{0} \in[V]$; let $a \leq \alpha<\beta<b$; then

$$
\begin{aligned}
& \int_{a}^{b} x_{(\alpha, \beta]}(t) T_{\circ}\left(\int_{a}^{t} f(s) d s\right) d t= \\
& -\int_{a}^{b}\left(\int_{a}^{s} x_{(\alpha, \beta]}(t) d t\right) T_{o} f(s) d s+(\beta-\alpha) T_{0} \int_{a}^{b} f(s) d s .
\end{aligned}
$$


Proof.

$$
\begin{aligned}
\int_{\beta}^{\alpha} T_{0}\left(\int_{\alpha}^{t} f(s) d s\right) d t & =\int_{\alpha}^{\beta}\left(\int_{\alpha}^{t}\left(T_{0} f\right)(s) d s\right) d t \quad \text { by [2], Theorem 3.7.12, } \\
& =\int_{\alpha}^{\alpha}\left(\int_{\alpha}^{\beta} T_{0} f(s) d t\right) d s+\int_{\alpha}^{\beta}\left(\int_{s}^{\beta} T_{0} f(s) d t\right) d s \text { by [2], } \\
& =\int_{a}^{\alpha}(\beta-\alpha) T_{0} f(s) d s+\int_{\alpha}^{\beta}(\beta-s) T_{0} f(s) d s ;
\end{aligned}
$$

which yields the right side of the stated result by rearrangement.

THEOREM B. If $f: I \rightarrow V$ and $T():. I \rightarrow[V]$ are Bochner-integrable on $I$, then

$$
\left.\left.\int_{I} T(t)\left(\int_{a}^{t} f(s) d s\right) d t=-\int_{I}\left(\int_{a}^{s} T(t) d t\right) f(s) d s+\iint_{I} T(t) d t\right) \iint_{I} f(s) d s\right) .
$$

REMARK. For each $t \in I, T(t)$ is a bounded linear map from $V$ into $V$. If, in particular, $V=R$, then $T(t) \int^{t} f(s) d s$ is of the form $\varphi(t) \int^{t} f(s) d s$, for some function $\varphi($.$) ; and the result reduces$ to the usual integration-by-parts formula. But in general, each integral is a Bochner integral on $I=[a, b]$ or a subinterval.

Proof. Lemma 1 gives the result, in case $T(t)=\chi_{(\alpha, \beta]}(t) T_{0}$ and $T_{0} \in[\mathrm{V}]$; hence Theorem B holds for any Bochner-integrable $f$ and any step-function $T($.$) , that is, any function T($.$) which assumes only$ finitely many values in $[V]$, each on a subinterval of $I$. In terms of the norm $\|T\|=\int_{I}\|T(t)\| d t$, the step-functions are a dense subspace of the Bochner-integrable functions; so Theorem $B$ follows, from the definition of Bochner integral.

Theorem $B$ has a variant in terms of line integrals in a (real) Banach space $V$, taken by convention along straight segments. If $a, b \in V, \beta$ is a real variable, $x=a+\beta b,\|b\|=1$, denote also $\int \ldots d|x|$ to 
mean $\int \ldots d \beta$ and $\int \ldots d x$ to mean $\int \ldots(d \beta) b$.

THEOREM C. Let $A$ and $V$ be real Banach spaces; $U$ a convex open subset of $A ; T(a) \in[V]$ for each $a \in U ; h: U \rightarrow V$ a continuous Fréchet-differentiable map such that, for $a, b \in A,\|b\|=1$, and $a, c=a+\lambda b \in U, h(\alpha+\beta b) b$ is an absolutely continuous function of $\beta \in[0, \lambda]$. Then

$$
\int_{a}^{c} T(x) h(x) d|x|=-\int_{a}^{c}\left(\int_{a}^{z} T(x) d|x|\right) h^{\prime}(z) d z+\left(\int_{a}^{c} T(x) d|x|\right) h(c) .
$$

Proof. From Theorem B,

$$
\begin{aligned}
& \int_{0}^{\lambda} T(a+\beta b)\left(\int_{0}^{\alpha} h^{\prime}(a+\alpha b) b d \alpha\right) d \beta= \\
& \quad-\int_{0}^{\lambda}\left(\int_{0}^{\alpha} T(a+\beta b) d \beta\right) h^{\prime}(a+\alpha b) b d \alpha+\left(\int_{0}^{\lambda} T(\alpha+\beta b) d \beta\right)\left(\int_{0}^{\lambda} h^{\prime}(a+\alpha b) b d \alpha\right) .
\end{aligned}
$$

Define $f:[0, \lambda] \rightarrow V$ by $f(\beta)=h(a+\beta b) b$; let $e \in V$; let $P$ be the projector of $V$ onto the one-dimensional subspace spanned by $e$; then $P f$ is absolutely continuous, mapping $[0, \beta]$ into $R e$; therefore

$$
(P f)(\alpha)-(P f)(0)=\int_{0}^{\alpha}(P f)^{\prime}(\beta) d \beta=P \int_{0}^{\alpha} f^{\prime}(\beta) d \beta \quad(0<\alpha<\lambda)
$$

since $h$, and therefore $f$, is Fréchet-differentiable. Therefore, since $e$ is arbitrary,

$$
h(\alpha+\alpha b)-h(a)=f(\alpha)-f(0)=\int_{0}^{\alpha} h^{\prime}(a+\beta b) b d \beta .
$$

Substitution of this expression into the result from Theorem B proves the theorem.

\section{References}

[1] B.D. Craven, "On the Gauss-Green theorem", J. Austral. Math. Soc. 8 (1968), 385-396. 
[2] Einar Hille and Ralph S. Phillips, Functional analysis and semi-groups (Colloquium Publ. 31, revised ed., Amer. Math. Soc., Providence, R.J., 1957).

[3] Kôsaku Yosida, Functional analysis, 2nd ed. (Die Grundlehren der mathematischen Wissenschaften, Bani 123, Springer-Verlag, Berlin, Heidelberg, New York, 1968).

University of Melbourne,

Parkville, victoria. 